\title{
Nocturnal enuresis is associated with moderate-to-severe obstructive sleep apnea in children with snoring
}

\author{
Emmanouel I. Alexopoulos ${ }^{1}$, Georgia Malakasioti' ${ }^{1}$ Vasiliki Varlami' ${ }^{1}$, Michail Miligkos ${ }^{2}$, Konstantinos Gourgoulianis ${ }^{1}$ and \\ Athanasios G. Kaditis ${ }^{1,2}$
}

\begin{abstract}
BACKGROUND: Conflicting data suggest that prevalence of monosymptomatic primary nocturnal enuresis (NE) increases with increasing severity of obstructive sleep apnea (OSA) in childhood and especially in girls. We hypothesized that NE is associated with increased risk of moderate-to-severe OSA (obstructive apnea-hypopnea index (AHI) $>5$ episodes/hour) among children with snoring.
\end{abstract}

METHODS: Data of children ( $\geq 5 \mathrm{y}$ old) with snoring who were referred for polysomnography over 12 y were reviewed.

RESULTS: Data of 525 children with mean age $( \pm$ SD) 7.5 ( \pm 2.2) y and median obstructive AHI (10th-90th percentiles) 1.9 (0.4-7.3) episodes/hour were analyzed. Three hundred and fifty-five children (67.6\%) had NE and 87 (16.6\%) had moderate-to-severe OSA. There was no interaction between NE and gender regarding the association with moderate-to-severe OSA $(P>0.05)$. NE was associated significantly with presence of moderate-to-severe OSA after adjustment for tonsillar hypertrophy, obesity, gender, and age (adjusted odds ratio = $1.92(1.08-3.43) ; P=0.03)$. Presence of NE had high sensitivity (78.2\%) and low positive predictive value (19.2\%) for detecting moderate-to-severe OSA and low specificity (34.5\%) and high negative predictive value (88.8\%) for ruling it out.

CONCLUSION: Children with snoring and without NE referred for polysomnography are less likely to have moderate-tosevere OSA compared to those with NE.

$\mathrm{H}$ abitual snoring is the most common clinical manifestation of obstructive sleep-disordered breathing (SDB) which ranges in severity from primary snoring to upper airway resistance, obstructive hypoventilation and obstructive sleep apnea (OSA) (1). Monosymptomatic primary nocturnal enuresis (NE) is considered one of the morbidities associated with SDB and its treatment by adenotonsillectomy or other therapeutic interventions such as nasal corticosteroids or rapid maxillary expansion is accompanied by resolution or reduced frequency of enuresis episodes (1-5).

Although habitual snoring has been associated with the presence of NE in population-based studies consistently, it is unclear whether a relationship exists between prevalence of enuresis and severity of $\operatorname{SDB}(6,7)$. It has been proposed that the prevalence of enuresis is not modified by the severity of respiratory and sleep disturbance (7), whereas other authors have suggested that it increases in parallel with OSA severity in girls only (8).

The majority of children with habitual snoring and adenotonsillar hypertrophy do not undergo a sleep study prior to adenoidectomy and/or tonsillectomy in order to assess OSA severity due to limited access to sleep laboratories with expertise in children (9). In this study, it was hypothesized that NE is a significant risk factor for finding an obstructive apneahypopnea index (AHI) $>5$ episodes/hour OSA in children with snoring who are referred for polysomnography by primary care physicians. Furthermore, the potential interaction between NE and female gender regarding the association with obstructive AHI $>5$ episodes/hour was explored. Obstructive AHI $>5$ episodes/hour corresponds to moderate-to-severe OSA as proposed by Dayyat et al. (10) and it is related to elevated systolic and diastolic blood pressure, increased risk of development of respiratory complications and persistent OSA following adenotonsillectomy as well as low likelihood of spontaneous OSA resolution without treatment interventions (11-14).

\section{RESULTS}

\section{Subjects' Characteristics}

A total of 1,058 children with history of snoring ( $\geq 1$ night/week) underwent polysomnography during the study period and 703 of them were $\geq 5 \mathrm{y}$ old. Data of 178 out of 703 subjects were excluded from the analysis due to history of craniofacial abnormalities $(n=52)$, neuromuscular or genetic disorders $(n=118)$, spina bifida $(n=2)$, daytime enuresis $(n=4)$ or diabetes mellitus $(n=2)$. The mean age $( \pm \mathrm{SD})$ of the remaining 525 children was $7.5 \pm 2.2 \mathrm{y}$ and the median obstructive AHI (10th-90th percentiles) was 1.9 episodes/hour (0.4-7.3 episodes/hour). Three hundred and fifty-five of the 525 included children (67.6\%) had NE and 87 (16.6\%) had moderate-to-severe OSA. None of the 525 included subjects had secondary enuresis. Secondary enuresis was present in four of the children with craniofacial abnormalities and in the two subjects with diabetes mellitus.

\footnotetext{
'Sleep Disorders Laboratory, University of Thessaly School of Medicine and Larissa University Hospital, Larissa, Greece; ${ }^{2}$ Pediatric Pulmonology Unit, Sleep Disorders Laboratory, First Department of Pediatrics, University of Athens School of Medicine and Aghia Sophia Children's Hospital, Athens, Greece. Correspondence: Athanasios G. Kaditis (kaditia@hotmail.com)
} 


\section{Articles | Alexopoulos et al.}

Children with NE did not differ from those without NE in terms of age, polysomnography indices or frequency of female subjects (Table 1). Nevertheless, the NE group had higher BMI $\mathrm{z}$ score and proportions of obese children and children with moderate-to-severe OSA compared to the group without NE (Table 1). Amongst children with NE $(n=355), 68(19.2 \%)$ had obstructive AHI >5 episodes/hour, 209 (58.8\%) had obstructive AHI of $1-5$ episodes/hour and 78 (22\%) had obstructive AHI $<1$ episode/hour. Of subjects without NE $(n=170), 19$ (11.2\%) had obstructive AHI $>5$ episodes/hour, 103 (60.6\%) had obstructive AHI of 1-5 episodes/hour, and 48 (28.2\%) had obstructive AHI <1 episode/hour.

\section{Association of Moderate-to-Severe OSA With NE}

$\mathrm{NE}$ was associated significantly with presence of moderateto-severe OSA: OR $(95 \% \mathrm{CI})=1.88(1.09-3.25) ; P=0.02$. In the first multivariate logistic regression model, the interaction of enuresis with gender regarding the association with moderate-to-severe OSA was not significant $(P>0.05)$. When the interaction term was removed from the multivariate logistic regression analysis model, the significant relationship between NE and moderate-to-severe OSA persisted after adjustment for the presence of tonsillar hypertrophy, obesity, gender, and age (adjusted OR $=1.92(1.08-3.43) ; P=0.03)$ (Table 2).

\section{Sensitivity, Specificity, Positive, and Negative Predictive Values of NE for Predicting or Ruling Out Obstructive AHI >5 Episodes/ Hour}

Presence of NE had high sensitivity (78.2\%) and low positive predictive value (19.2\%) for detecting moderate-to-severe OSA along with low specificity (34.5\%) and high negative predictive value $(88.8 \%)$ for ruling it out.

\section{DISCUSSION}

In this large study of children with snoring and age $\geq 5 \mathrm{y}$ who were referred for polysomnography by primary care

Table 1 Subjects' characteristics and statistical comparisons of children with and without nocturnal enuresis (NE)

\begin{tabular}{|c|c|c|c|}
\hline Variables & Children with NE $(n=355)$ & Children without NE $(n=170)$ & $P$ value \\
\hline Age, years & $7.6 \pm 2.3$ & $7.3 \pm 2.1$ & NS \\
\hline Gender, female (\%) & $154(43.4)$ & $70(41.2)$ & NS \\
\hline Obesity (\%) & $123(34.6)$ & $16(9.4)$ & $<0.01$ \\
\hline Tonsillar hypertrophy (>2+) (\%) & $228(64.2)$ & $118(69.4)$ & NS \\
\hline Respiratory arousal index, episodes/hour & $0.8(0.1-4)$ & $0.8(0-2.9)$ & NS \\
\hline Oxygen desaturation ( $\geq 4 \%$ ) of hemoglobin index, episodes/hour & $2.3(0.5-10.9)$ & $2.5(0.5-9.8)$ & NS \\
\hline Oxygen saturation of hemoglobin nadir, $\%$ & $90(84-93)$ & $90(84-93)$ & NS \\
\hline Subjects with apnea-hypopnea index $>5$ episodes/hour, $\%$ & $68(19.2)$ & $19(11.2)$ & $<0.05$ \\
\hline
\end{tabular}

Continuous variables are expressed as mean \pm SD or median (10th-90th percentiles).

Table 2 Associations of moderate-to-severe OSA (obstructive AHI $>5$ episodes/hour) with history of NE and other variables

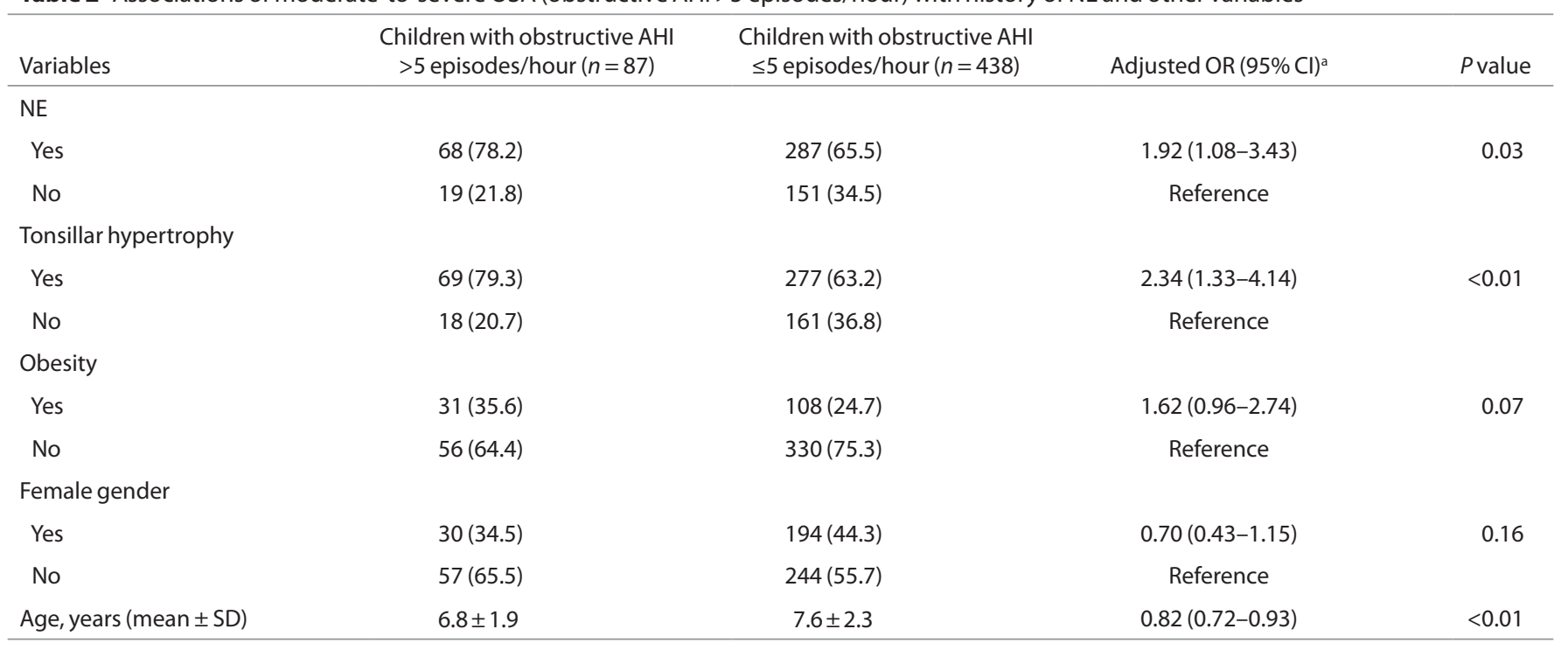

AHI, apnea-hypopnea index; Cl, confidence interval; NE, nocturnal enuresis; OR, odds ratio; OSA, obstructive sleep apnea. 
physicians, it was shown that NE was significantly associated with the presence of obstructive AHI $>5$ episodes/hour in polysomnography. The association between NE and moderateto-severe OSA did not differ between boys and girls. Children with habitual snoring and without NE who are referred for further evaluation are less likely to have moderate-to-severe of OSA compared to those with NE.

Nocturnal enuresis is the involuntary loss of urine while sleeping in children $\geq 5 \mathrm{y}$ old, the age when urinary continence is expected at night $(15,16)$. The enuresis is characterized as primary in the absence of a previous dry period of at least 6 mo and monosymptomatic if there are no other lower urinary tract symptoms or history of bladder dysfunction (16). The minimum frequency of nocturnal urine loss that has been used to define NE in various studies has ranged from once in the last year to twice a week $(15,17)$.

Snoring is the commonest clinical manifestation of SDB and its presence is clearly associated with increased risk of $\mathrm{NE}$ in childhood (6,7). In a population-based cohort of 5-14 y-old children residing in Central Greece, the prevalence of NE (urinary incontinence for at least one night per week) was $7.4 \%$ in subjects with habitual snoring and $2 \%$ in children without snoring (6). In a similar cohort from the United States, the frequency of enuresis (urinary incontinence at least two to six times per month) in 5-7 y-old children was $26.9 \%$ in habitual snorers versus $11.6 \%$ in non-habitual snorers (7). Much higher prevalence of NE (41\%) has been reported in children with SDB referred for polysomnography (18).

The clinically significant correlation between intermittent upper airway obstruction during sleep and enuresis is further supported by a systematic review of seven studies including 1,360 children with SDB indicating reduction in the frequency of enuresis after AT (2). Several different pathophysiologic mechanisms have been proposed to explain the association between the two conditions. An increase in intra-abdominal pressure caused by intense inspiratory efforts against a closed upper airway has been implicated in the pathogenesis of enuresis (19). In addition, we have previously shown that fractional urinary excretion of sodium increases in parallel with severity of SDB and diastolic blood pressure in children with snoring (20).

At the termination of nocturnal obstructive events, repetitive increases in systemic blood pressure occur which may lead to pressure-induced natriuresis $(21,22)$. Furthermore, upper airway obstruction is associated with exaggerated negative intrathoracic pressure swings, increased systemic venous return and preload to the right ventricle along with increased left cardiac ventricle after load $(23,24)$. Acute overload of the cardiac ventricles could promote release of brain natriuretic peptide from ventricular myocytes which induces vasodilation and natriuresis (25). Adenotonsillectomy for OSA in childhood is accompanied by reduction of morning brain natriuretic peptide levels in venous blood (26). However, future studies should assess whether adenotonsillectomy is followed by decrease in both urine sodium excretion and nocturnal urine production.
Although snoring is a risk factor for NE, it is still unclear whether SDB severity affects the prevalence of NE. In the US cohort, children with habitual snoring and enuresis did not differ from subjects with habitual snoring and without enuresis regarding AHI (7). Other authors concluded that among children referred for polysomnography there was no progressive increase in the frequency of enuresis with increasing AHI (18). However, in a hospital-based, case-control study, NE was significantly related to OSA severity (27). Furthermore, Chinese children with and without OSA recruited from the community were similar regarding frequency of enuresis $(\geq 1$ night/ month), but the frequency increased with increasing severity of OSA in girls only (8).

Diagnosis of moderate-to-severe OSA is important because affected patients can develop respiratory complications or may have persistent disease following AT (12-14). In the current large cohort of hospital-referred children with habitual snoring, NE was significantly associated with the presence of moderate-to-severe OSA irrespective of gender. Additionally, the great majority of snorers without $\mathrm{NE}$ had only mild OSA. Hence, in health systems with limited resources, nonenuretic children who snore are of low priority for full nocturnal polysomnography.

\section{Conclusion}

Amongst children with snoring who are referred for polysomnography by primary care physicians, those without NE are less likely to have moderate-to-severe OSA relative to subjects with NE.

\section{METHODS}

\section{Subjects and Evaluation}

We retrospectively analyzed archived data of children with snoring ( $\geq 1$ night/week) and age $\geq 5$ y who were referred for polysomnography to the Larissa University Hospital by primary care physicians from January 2001 till December 2012. Data of subjects with craniofacial abnormalities, neuromuscular or genetic disorders, spina bifida, lower urinary tract symptoms other than enuresis (e.g., daytime incontinence, urgency, hesitancy, straining) and history of bladder dysfunction, diabetes mellitus or diabetes insipidus were not considered in the analysis.

Prior to polysomnography, parents underwent an interview including questions related to their child's health and more specifically: symptoms of SDB, presence and frequency of enuresis and history of any chronic disease. Frequency of bedwetting was graded as (i) every night; (ii) at least one night per week but not every night; (iii) at least one night per month but not every week; or (iv) never (6). All referred children were examined, height and body weight were measured, BMI z score was calculated and tonsillar size was graded visually using a score with values from $1+$ to $4+(28,29)$. Physical examination in all children was completed by one of the investigators (E.I.A.).

\section{Polysomnography}

Alice 4 or 5 computerized systems (Healthdyne, Marietta, GA) were used for overnight polysomnography and the following signals were recorded: electroencephalogram (C3/A2, C4/A1, O1/A2); right and left oculogram; submental and tibial electromyogram; body position; electrocardiogram; thoracic and abdominal wall motion (piezoelectric transducers); oronasal airflow (3-pronged thermistor and nasal pressure transducer); and oxygen saturation of hemoglobin. 
Obstructive apnea was scored when chest/abdominal wall motion was detected in the absence of airflow for at least two breaths in duration $(30,31)$. Apnea was considered of mixed type when there was both an obstructive and a central component. Hypopnea was defined as: (i) a reduction in the airflow signal amplitude of at least 50\% compared to baseline; and (ii) associated with oxygen desaturation of hemoglobin equal to or greater than $4 \%$ or with an arousal. Obstructive AHI was the mean number of obstructive and mixed apneas and hypopneas per hour of total sleep time (13). Arousals and sleep stages were evaluated according to standard criteria $(32,33)$. Polysomnography scoring in all children was completed by the same investigator (E.I.A.) and reviewed by a second investigator (A.G.K.). Beginning in June 2009, the new American Academy of Sleep Medicine criteria for the scoring of sleep and associated events were implemented in the Larissa University Hospital Sleep Disorders Laboratory (34). For the purposes of this study and for consistent data analysis, sleep studies performed in June 2009 or more recently were re-scored according to the old rules (30-33).

\section{Data Analysis}

Obstructive AHI $>5$ episodes/hour was the main outcome variable of the study (7). NE was the primary explanatory variable and was defined as intermittent urine incontinence while sleeping for at least one night per week in the absence of (i) other lower urinary tract symptoms (increased/decreased voiding frequency, daytime incontinence, urgency, hesitancy, straining, weak stream, intermittency, holding maneuvers, feeling of incomplete emptying, post-micturition dribble, and genital or lower urinary tract pain); (ii) history of bladder dysfunction; or (iii) a previous dry period of at least 6 mo $(16,35)$. The cut-off value of at least one night per week was selected for consistency with previous population-based epidemiologic studies $(6,7)$. When there was a previous dry period of at least $6 \mathrm{mo}$, the term secondary enuresis was applied. Tonsillar hypertrophy (tonsillar size $>2+$ ), obesity i.e., BMI greater than the 95th percentile for age and gender (BMI z score $>1.645$ ), gender and age were other explanatory variables that may be related to OSA severity and were also considered in the analysis.

Children with and without NE were compared in terms of age, gender, BMI z score, polysomnography indices and frequencies of tonsillar hypertrophy, obesity and percentage of subjects with obstructive AHI $>5$ episodes/hour. Student's $t$-test was carried out for continuous variables that approached a normal distribution, Mann-Whitney $U$-test was used for continuous variables that did not follow a normal distribution, and $\chi^{2}$ was applied for categorical characteristics.

Odds ratios (OR) and the corresponding 95\% confidence intervals (CI) for obstructive AHI $>5$ episodes/hour in children with versus without NE were calculated using univariate logistic regression analysis. Multivariate logistic regression analysis was completed in order to adjust OR for NE by all explanatory variables including the interaction of enuresis with gender. Multivariable analysis was repeated retaining only the explanatory variables without the interaction.

Sensitivity, specificity, positive, and negative predictive values of NE for prediction of moderate-to-severe OSA were calculated. The analysis was completed by SPSS 17.0; SPSS, Chicago, IL). This retrospective research protocol was approved by the Larissa University Hospital Ethics Committee and informed consent was not necessary to use these patients' data retrospectively.

Disclosure: There is no conflict of interest to disclose.

\section{REFERENCES}

1. Kaditis A, Kheirandish-Gozal L, Gozal D. Algorithm for the diagnosis and treatment of pediatric OSA: a proposal of two pediatric sleep centers. Sleep Med 2012;13:217-27.

2. Jeyakumar A, Rahman SI, Armbrecht ES, Mitchell R. The association between sleep-disordered breathing and enuresis in children. Laryngoscope 2012;122:1873-7.

3. Alexopoulos EI, Kaditis AG, Kostadima E, Gourgoulianis K. Resolution of nocturnal enuresis in snoring children after treatment with nasal budesonide. Urology 2005;66:194.

4. Schütz-Fransson U, Kurol J. Rapid maxillary expansion effects on nocturnal enuresis in children: a follow-up study. Angle Orthod 2008;78:201-8.
5. Kovacevic L, Jurewicz M, Dabaja A, et al. Enuretic children with obstructive sleep apnea syndrome: should they see otolaryngology first? J Pediatr Urol 2013;9:145-50.

6. Alexopoulos EI, Kostadima E, Pagonari I, Zintzaras E, Gourgoulianis K, Kaditis AG. Association between primary nocturnal enuresis and habitual snoring in children. Urology 2006;68:406-9.

7. Sans Capdevila O, Crabtree VM, Kheirandish-Gozal L, Gozal D. Increased morning brain natriuretic peptide levels in children with nocturnal enuresis and sleep-disordered breathing: a community-based study. Pediatrics 2008;121:e1208-14.

8. Su MS, Li AM, So HK, Au CT, Ho C, Wing YK. Nocturnal enuresis in children: prevalence, correlates, and relationship with obstructive sleep apnea. J Pediatr 2011;159:238-42.e1.

9. Mitchell RB, Pereira KD, Friedman NR. Sleep-disordered breathing in children: survey of current practice. Laryngoscope 2006;116:956-8.

10. Dayyat E, Kheirandish-Gozal L, Gozal D. Childhood Obstructive Sleep Apnea: One or Two Distinct Disease Entities? Sleep Med Clin 2007;2: $433-44$.

11. Amin R, Somers VK, McConnell K, et al. Activity-adjusted 24-hour ambulatory blood pressure and cardiac remodeling in children with sleep disordered breathing. Hypertension 2008;51:84-91.

12. Nixon GM, Kermack AS, Davis GM, Manoukian JJ, Brown KA, Brouillette RT. Planning adenotonsillectomy in children with obstructive sleep apnea: the role of overnight oximetry. Pediatrics 2004;113(1 Pt 1):e19-25.

13. Bhattacharjee R, Kheirandish-Gozal L, Spruyt K, et al. Adenotonsillectomy outcomes in treatment of obstructive sleep apnea in children: a multicenter retrospective study. Am J Respir Crit Care Med 2010;182:676-83.

14. Marcus CL, Moore RH, Rosen CL, et al.; Childhood Adenotonsillectomy Trial (CHAT). A randomized trial of adenotonsillectomy for childhood sleep apnea. N Engl J Med 2013;368:2366-76.

15. Byrd RS, Weitzman M, Lanphear NE, Auinger P. Bed-wetting in US children: epidemiology and related behavior problems. Pediatrics 1996;98(3 Pt 1):414-9.

16. Nevéus T, von Gontard A, Hoebeke P, et al. The standardization of terminology of lower urinary tract function in children and adolescents: report from the Standardisation Committee of the International Children's Continence Society. J Urol 2006;176:314-24.

17. Gümüs B, Vurgun N, Lekili M, Iscan A, Müezzinoglu T, Büyuksu C. Prevalence of nocturnal enuresis and accompanying factors in children aged 7-11 years in Turkey. Acta Paediatr 1999;88:1369-72.

18. Brooks LJ, Topol HI. Enuresis in children with sleep apnea. J Pediatr 2003;142:515-8.

19. Arai H, Furuta H, Kosaka K, et al. Polysomnographic and urodynamic changes in a case of obstructive sleep apnea syndrome with enuresis. Psychiatry Clin Neurosci 1999;53:319-20.

20. Kaditis AG, Alexopoulos EI, Evangelopoulos K, et al. Correlation of urinary excretion of sodium with severity of sleep-disordered breathing in children: a preliminary study. Pediatr Pulmonol 2010;45:999-1004.

21. O’Driscoll DM, Foster AM, Ng ML, et al. Acute cardiovascular changes with obstructive events in children with sleep disordered breathing. Sleep 2009;32:1265-71.

22. Staessen JA, Birkenhäger W, Bulpitt CJ, et al. The relationship between blood pressure and sodium and potassium excretion during the day and at night. J Hypertens 1993;11:443-7.

23. Garpestad E, Parker JA, Katayama H, et al. Decrease in ventricular stroke volume at apnea termination is independent of oxygen desaturation. J Appl Physiol 1994;77:1602-8.

24. Bonsignore MR, Marrone O, Romano S, Pieri D. Time course of right ventricular stroke volume and output in obstructive sleep apneas. Am J Respir Crit Care Med 1994;149:155-9.

25. Yoshimura M, Yasue H, Morita E, et al. Hemodynamic, renal, and hormonal responses to brain natriuretic peptide infusion in patients with congestive heart failure. Circulation 1991;84:1581-8.

26. Kaditis AG, Chaidas K, Alexopoulos EI, Varlami V, Malakasioti G, Gourgoulianis $\mathrm{K}$. Effects of adenotonsillectomy on R-R interval and brain natriuretic peptide levels in children with sleep apnea: a preliminary report. Sleep Med 2011;12:646-51. 
27. Barone JG, Hanson C, DaJusta DG, Gioia K, England SJ, Schneider D. Nocturnal enuresis and overweight are associated with obstructive sleep apnea. Pediatrics 2009;124:e53-9.

28. Ogden CL, Kuczmarski RJ, Flegal KM, et al. Centers for Disease Control and Prevention 2000 growth charts for the United States: improvements to the 1977 National Center for Health Statistics version. Pediatrics 2002;109:45-60.

29. Brodsky L. Modern assessment of tonsils and adenoids. Pediatr Clin North Am 1989;36:1551-69.

30. American Thoracic Society. Standards and indications for cardiopulmonary sleep studies in children. Am J Respir Crit Care Med 1996;153:866-78.

31. Kaditis AG, Finder J, Alexopoulos EI, et al. Sleep-disordered breathing in 3,680 Greek children. Pediatr Pulmonol 2004;37:499-509.
32. Rechtschaffen A, Kales A. A Manual of Standardized Terminology: Techniques and Scoring Systems for Sleep Stages of Human Subjects. Los Angeles, CA: UCLA Brain Information Service/Brain Research Institute, 1968.

33. American Sleep Disorders Association. EEG arousals: scoring rules and examples: a preliminary report from the Sleep Disorders Atlas Task Force of the American Sleep Disorders Association. Sleep 1992;15:173-84.

34. Iber C, Ancoli-Israel S, Chesson A, Quan SF; American Academy of Sleep Medicine. The AASM Manual for the Scoring of Sleep and Associated Events: Rules, Terminology and Technical Specifications, 1st edition. Westchester, IL: American Academy of Sleep Medicine, 2007.

35. Hjalmas K, Arnold T, Bower W, et al. Nocturnal enuresis: an international evidence based management strategy. J Urol 2004;171(6 Pt 2):2545-61. 